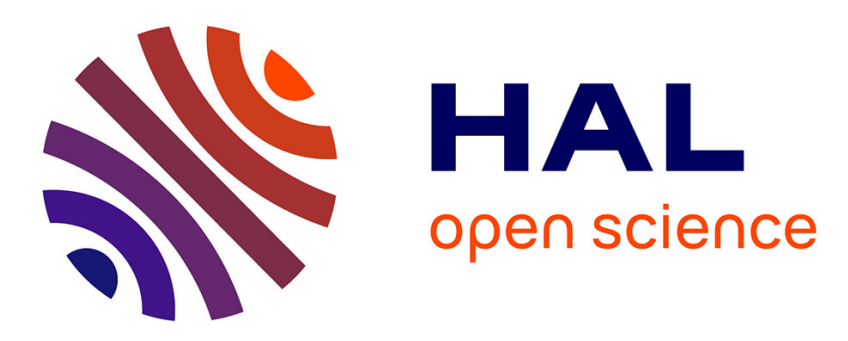

\title{
Accurate deuterium spectroscopy and comparison with ab initio calculations
}

\author{
S. Wojtewicz, R. Gotti, D. Gatti, M. Lamperti, P. Laporta, H. Jozwiak, \\ Franck Thibault, P. Wcislo, M. Marangoni
}

\section{- To cite this version:}

S. Wojtewicz, R. Gotti, D. Gatti, M. Lamperti, P. Laporta, et al.. Accurate deuterium spectroscopy and comparison with ab initio calculations. Physical Review A, 2020, 101 (5), pp.052504. 10.1103/PhysRevA.101.052504 . hal-02796937

\section{HAL Id: hal-02796937 \\ https://hal.science/hal-02796937}

Submitted on 18 Jun 2020

HAL is a multi-disciplinary open access archive for the deposit and dissemination of scientific research documents, whether they are published or not. The documents may come from teaching and research institutions in France or abroad, or from public or private research centers.
L'archive ouverte pluridisciplinaire HAL, est destinée au dépôt et à la diffusion de documents scientifiques de niveau recherche, publiés ou non, émanant des établissements d'enseignement et de recherche français ou étrangers, des laboratoires publics ou privés. 


\title{
Accurate deuterium spectroscopy and comparison with ab initio calculations
}

\author{
S. W'ojtewicz, ${ }^{1}$ R. Gotti, ${ }^{2}$ D. Gatti, ${ }^{2}$ M. Lamperti, ${ }^{2}$ P. Laporta, ${ }^{2}$ \\ H. J'o' zwiak, ${ }^{1}$ F. Thibault, ${ }^{3}$ P. Wcis- lo, and M. Marangoni ${ }^{2}$ \\ ${ }^{1}$ Institute of Physics, Faculty of Physics, Astronomy and Informatics, \\ Nicolaus Copernicus University in Torun', Grudziadzka 5, 87-100 Torun, Poland \\ ${ }^{2}$ Physics Department of Politecnico di Milano and IFN-CNR, Via Gaetano Previati 1/C, Lecco 23900, \\ Italy Univ Rennes, CNRS, IPR (Institut de Physique de Rennes)-UMR 6251, F-35000 Rennes, \\ France (Dated: March 16, 2020)
}

\begin{abstract}
We present accurate measurements of the quadrupole $\mathrm{S}(3)$ and $\mathrm{S}(4)$ transitions of the $\mathrm{D}_{2} 2-0$ band. Self-perturbed spectra were collected in a wide pressure range with a cavity ring-down spectrometer linked to an optical frequency comb. The results of ab initio quantum scattering calculations were incorporated into the line-shape analysis which was performed using the Hartmann-Tran profile. This approach allowed us to mitigate the collisional systematics and to reach a $900 \mathrm{kHz}$ accuracy of the transition frequencies, which represents a 30 -fold improvement compared to previously available data. We also compare our findings with newly calculated transition frequencies that include relativistic and QED corrections up to $\alpha^{7}$. The differences between theoretical and experimental values for the transition frequencies are $2.2 \mathrm{MHz}$ and $3.3 \mathrm{MHz}$ for the $\mathrm{S}(3)$ and $\mathrm{S}(4)$ transitions, respectively.

PACS numbers: Valid PACS appear here
\end{abstract}

\section{INTRODUCTION}

The energy levels structure of molecular hydrogen, the simplest neutral molecule, may be studied by theoreticians in an almost approximation-free approach [1]. Theoretical determination of rovibrational transitions energies of molecular hydrogen can nowadays reach the level of $2 \times 10^{-5} \mathrm{~cm}^{-1}[2,3]$ while the leading nonrelativistic energies can be accurate to $10^{-8} \mathrm{~cm}^{-1}[4,5]$. Such predictions allow for testing quantum electrodynamics (QED) for molecules at an unprecedented level of accuracy, also paving the way to the search of new physics beyond the Standard Model [6], such as extra dimensions [7] or fifth force [8].

Molecular hydrogen poses, however, a problem from the experimental point of view. Due to the symmetry of its electronic ground state the dipole transitions are forbidden (or are weak in case of HD), so that very weak electric quadrupole transitions must be handled. Moreover, in the case of molecular hydrogen the line shapes are atypical and difficult to model due to very pronounced collisional effects $[9,10]$. In the Doppler regime measurements of transition frequencies are complicated due to a number of line-shape effects contributing to the line asymmetry [11] that prevent a straightforward extrapolation of line centers to zero pressure, as needed for a comparison with theoretical determinations on the isolated molecule.

In this paper we present the most accurate up to date experimental determination of the frequencies of the $\mathrm{D}_{2} 2$ $-0 \mathrm{~S}(3)$ and $\mathrm{S}(4)$ transitions lying around $1.57 \mu \mathrm{m}$. The spectra were measured in a wide pressure range us-ing a cavity ring-down spectrometer linked to an optical frequency comb. The line-shape analysis was performed using the computationally cost-effective Hartmann-Tran profile $[12,13]$ modified for the so called $\beta$ correction
$[14,15]$, which was shown to be capable of describing molecular hydrogen line shapes [14]. The uncertainty of our measured line positions is $900 \mathrm{kHz}$, which represents a 30 -fold improvement compared to previously available data [16]. In order to reach this level of accuracy and to mitigate the collisional systematics we made use of $a b$ initio quantum scattering calculations on a highly-accurate potential energy surface to describe the collisional lineshape effects. We also validate our results against very recent theoretically determined transition frequencies $[2,3]$, which are characterized by a sub- $\mathrm{MHz}$ uncertainty and take into account relativistic and QED corrections up to $\alpha^{7}$.

\section{HIGH-FINESSE CAVITY-ENHANCED SPECTROMETER}

Figure 1 shows the layout of the spectrometer. Absolute calibration of the frequency axis is provided by a $100 \mathrm{MHz}$ Er:fiber femtosecond oscillator (Toptica FFS), whose repetition-rate $\left(f_{\text {rep }}\right)$ is phase-locked to a low-noise radiofrequency $(\mathrm{RF})$ oscillator referenced to a $10 \mathrm{MHz}$ GPS-disciplined Rubidium clock. The output of the oscillator seeds two amplifier stages delivering $220 \mathrm{~mW}$ of power at $1.55 \mu \mathrm{m}$ : one seeds the measurement branch of the comb through a dispersion-shifted telecom fiber which broadens the spectrum from 1.4 to $1.7 \mu \mathrm{m}$, cov-ering the region where the probe laser emits; the other drives the self-referencing arm of the comb, composed of a highly nonlinear fiber for supercontinuum generation and of an $\mathrm{f}-$ $2 \mathrm{f}$ interferometer for extraction of the carrier-envelopeoffset frequency $\left(f_{\text {ceo }}\right)$ of the comb [17] (not shown in the figure). The revealed $f_{\text {ceo }}$ is then band-pass filtered and frequency-locked against the Rubidium clock by a proportional-integrative-derivative (PID) servo. 


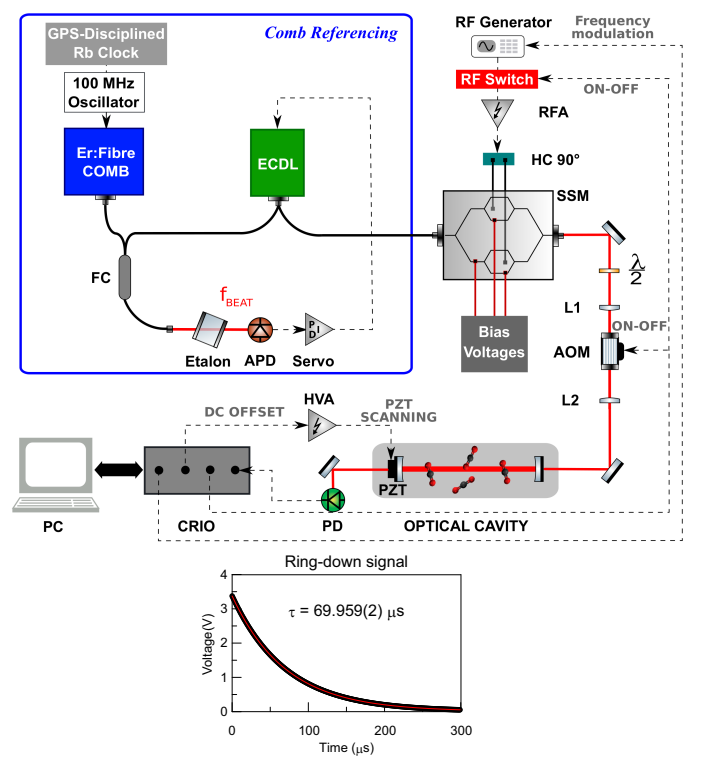

FIG. 1: Scheme of experimental setup. AOM: acousto-optical modulator; PZT: piezoelectric transducer; FC: fiber coupler; PD: photodetector; APD: avalanche photodetector; HVA: high voltage amplifier; $\mathrm{HC} 90^{\circ}$ : hybrid $90^{\circ}$ coupler; SSM: single-sideband modulator; PC: personal computer; L1, L2: lenses; CRIO: compact reconfigurable input/output board; ECDL: external cavity diode laser; RFA: radio-frequency amplifier; $\mathrm{f}_{\mathrm{BEAT}}$ : beat-note frequency between comb and ECDL. Black, red, and dashed-gray lines refer to fibers, free-space laser paths, and electrical signals, respectively. The bottom panel shows a typical ring-down decay signal observed in the experiment.

The probe laser is an external cavity diode laser (ECDL, Toptica DL pro) with an average emission power of about $25 \mathrm{~mW}$ from 1.5 to $1.63 \mu \mathrm{m}$, whose output is split in two parts. A $10 \%$ fraction is superimposed to the measurement arm of the comb and generates the beat note signal for the frequency locking of the laser to the comb, via feedback control of the laser grating position. The remaining $90 \%$ is coupled to a single-sideband modulator (SSM) that enables agile tuning of one of the sidebands over a range up to $20 \mathrm{GHz}$ while suppressing carrier and mirror sideband by 50 and $35 \mathrm{~dB}$, respectively [18]. The SSM is driven by an RF synthesizer (Anapico ASIN12G) followed by a broadband amplifier providing a $29 \mathrm{dBm}$ power from 1 to $12 \mathrm{GHz}$, which corresponds to the effective agile tuning range in the optical domain. To compensate for the $10 \%$ modulation efficiency of the SSM, the single-sideband undergoes optical amplification up to $20 \mathrm{~mW}$ by means of a broadband semiconductor optical amplifier (Thorlabs BOA1004S)

The single sideband seeds a $50 \mathrm{~cm}$-long stainless-steel high finesse cavity $(\mathcal{F}>125000)$ housed in a wooden box and tightened between an aluminum shell, to im-prove its mechanical stability and thermal conductiv-ity. The temperature of the shell is actively kept at $27.5^{\circ} \mathrm{C}$ by silicone-rubber stripe heaters. A real-time sys-
TABLE I: Uncertainty budget split into statistical (A) and systematic (B) contributions for the line positions $\left(\nu_{0}\right)$ of the $\mathrm{D}_{2} 2-0 \mathrm{~S}(3)$ and $\mathrm{S}(4)$ transitions. All quoted values are $1 \sigma$ uncertainties.

\begin{tabular}{l|r|r}
\hline & $\mathbf{S}(3)$ & $\mathbf{S}(4) \mathrm{u}\left(\nu_{0}\right)$ \\
\hline Uncertainty source (type) Statistics & $(\mathrm{kHz}) \mathrm{u}\left(\nu_{0}\right)$ & $\mathrm{kHz})$ \\
\hline (A) & 390 & 404 \\
Line-shape analysis (B) & 777 & 913 \\
Temperature instability (B) Etalons & 11 & 12 \\
(B) & - & 32 \\
Pressure gauge nonlinearity (B) & 35 & 39 \\
Frequency axis (B) & 6 & 6 \\
Standard combined & $\mathbf{8 7 0}$ & $\mathbf{9 9 9}$ \\
\hline
\end{tabular}

uncertainty

tem equipped with a Virtex-5 LX50 Field-Programmable Gate Array (FPGA, depicted as CRIO in Fig. 1) provides a signal to the RF synthesizer for frequency modulation of the single sideband (amplitude $\pm 4 \mathrm{MHz}$, period $3 \mathrm{~ms}$ ) to periodically bring it in coincidence with the cavity resonance. The same board also delivers a tracking signal to the cavity piezoelectric transducer (PZT) to accommodate cavity length drifts that might bring the cavity resonance outside the frequency modulation window of the single sideband. The ring-down traces at the cav-ity output are measured by an InGaAs detector with a gain of $10^{6} \mathrm{~V} / \mathrm{W}$ and $300 \mathrm{kHz}$ bandwidth. Every time a threshold of about $3 \mathrm{~V}$ is overcome at the cavity output, the SSM and also an acousto-optic modulator (AOM) placed before the cavity are simultaneously switched off to start the ring-down event. Their combined use turns out to be useful to suppress distortions of the exponen-tial decays. The decay constants are extracted by the FPGA-board through a real-time fitting algorithm [19]. To compensate for the Doppler shift effect due to the fre-quency modulation of the single sideband over the cavity resonance, every spectral point is the average of an even number of ring-downs with opposite tuning directions.

Optical spectra are acquired by a frequency-agile rapidscanning (FARS) approach [20] for the single side-band [21]: when a predefined number of decay constants is acquired for a given spectral point, the SSM increases the single-sideband frequency by a cavity free spectral range (FSR) to acquire other decay constants. Once the selected frequency span is completed, the laser fre-quency is slowly shifted by $50 \mathrm{MHz}$ via SSM, allowing the feedback loop acting on the PZT to modify the cav-ity length accordingly. A second series of FSR-spaced spectral points is then acquired in the reverse direction over the same span and so on. The final spectrum, ob-tained by interleaving of back and forth scans, is char-acterized by a spacing of $50 \mathrm{MHz}$ between consecutive spectral points. 

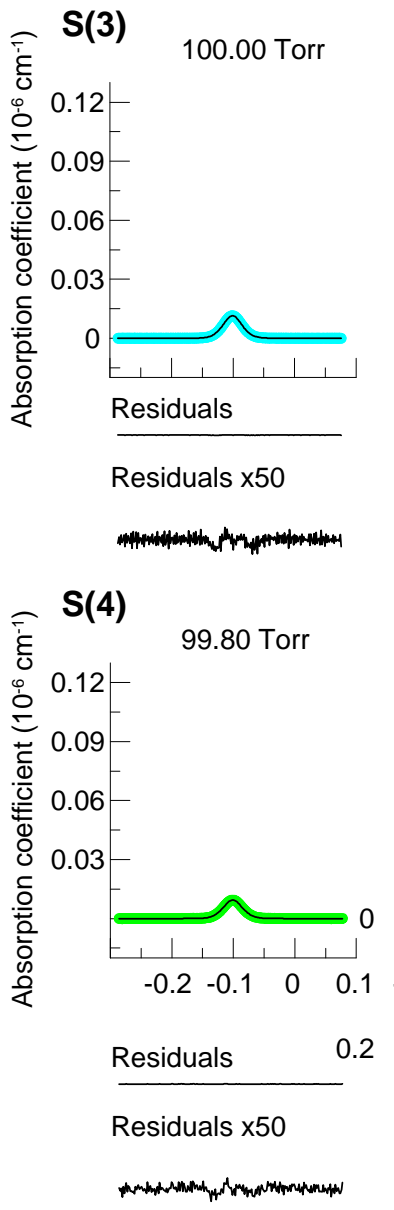
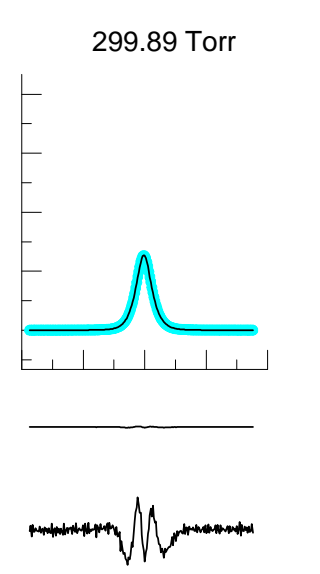

299.93 Torr
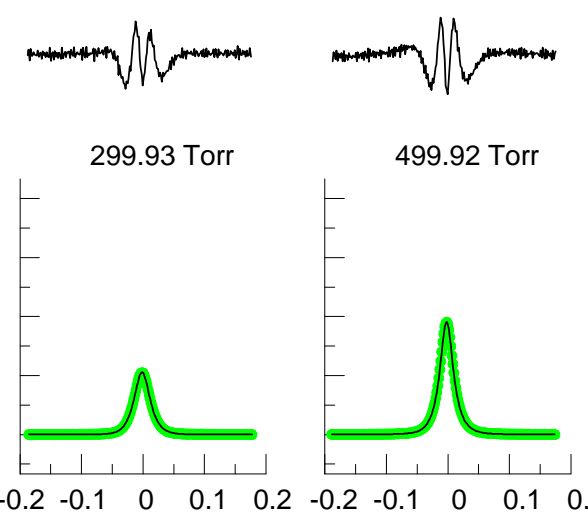

499.92 Torr
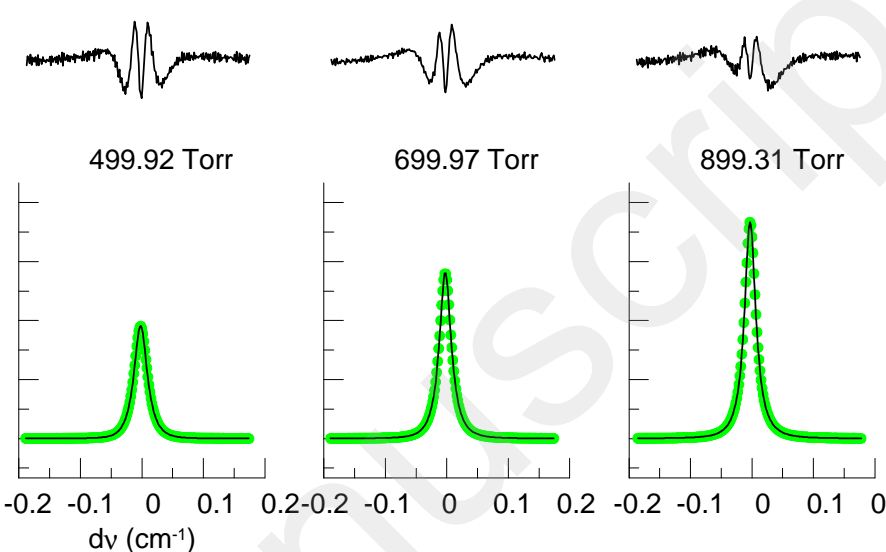

899.31 Torr

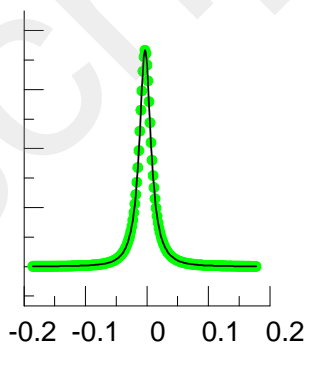

FIG. 2: Measured (dots) and fitted (solid lines) spectra of the $\mathrm{D}_{2} 2-0 \mathrm{~S}(3)$ and $\mathrm{S}(4)$ transitions and residuals from the HTP multispectrum fits. Each spectrum is an average of 200 scans.

\section{EXPERIMENTAL DETERMINATION OF THE ROVIBRATIONAL ENERGIES}

The $\mathrm{D}_{2}$ spectra were measured at five pressures of about 100 Torr, 300 Torr, 500 Torr, 700 Torr, and 900 Torr (see Fig. 2 for exact values) and at a tem-perature of $300.65(2)$ $\mathrm{K}$. The recorded spectra, presented in Fig. 2, are characterized by a signal-to-noise ratio (at the highest pressures) of about 5000. The used deuterium sample purity is $99.96 \%$. The accuracy of the pressure gauge is $0.15 \%$.

In order to fit the collected $\mathrm{D}_{2}$ spectra we chose the Hartmann-Tran profile (HTP) [12, 13]. Apart from the simplest physical effect affecting the line shape, i.e. the Doppler broadening and the collisional broadening and shifting, this model includes other effects such as the velocity-changing collisions leading to Dicke narrowing [22], the speed-dependence of collisional broadening and shifting [23], and the correlations between velocity- and phase-changing collisions [24]. The HTP uses a quadratic model describing the speed-dependent effects as given in Ref. [25] and the changes of the molecules' velocities are described through the hard-collision model [26]. This profile has very low computational cost and was recommended by the IUPAC as a reference model to describe spectra of atmospheric gases [27] while, after inclusion of the so called $\beta$ correction $[14,15]$, was shown to be also capable of describing molecular hydrogen line shapes [14]. To mitigate the collisional systematics, i.e. to reduce the correlations between fitted line-shape parameters and their systematic errors, we simultaneously applied two strategies. On the one hand we used a multispectrum fitting technique [23] in which a set of spectra recorded at multiple pressures is fitted simultaneously to enforce the right physical behavior of the parameters (e.g. a lin-ear dependence of the collisional width on pressure). On the other hand, to describe the various collisional effects on the line shapes we calculated the relevant generalized cross sections [29, 30]. They were deduced from scattering matrix elements obtained by performing quantum dynamics on the $\mathrm{D}_{2}-\mathrm{D}_{2}$ potential energy surface (PES) of Ref. [31]. In the analysis we fixed the speed dependence of the collisional broadening and shift $\left(\gamma_{S D}, \delta_{S D}\right)$ as well as the real part of the Dicke narrowing $\left(\nu_{o}^{r} p t\right)$ to the $a b$ 
initio values. The remaining parameters (line position $\nu_{0}$, collisional width $\gamma$ and shift $\delta$, and imaginary part of the Dicke narrowing $\nu_{p t}^{i}$ ) were fitted. The line areas and the baseline parameters including an etalon (for the $\mathrm{S}(4)$ transition, see next paragraph) were instead fitted individually for each pressure. The line positions retrieved from this fitting procedure are $6335.717325(29) \mathrm{cm}^{-1}$ and $6423.966880(33) \mathrm{cm}^{-1}$ for the $\mathrm{S}(3)$ and $\mathrm{S}(4)$ tran-sitions, respectively (see also Table II). The residuals of the multispectrum fits are presented in Fig. 2. A slight asymmetry emerges at the highest pressures, which we would have avoided by fitting all line-shape parameters. However, such approach was discarded since it may easily lead to spectroscopic parameters with poor physical meaning, as well as with bigger uncertainty due to the strong correlations between them. A detailed discussion about the impact of the numerical correlations between line-shape parameters on the determination of a transition frequency in the case of molecular hydrogen is given in Ref. [30].

The most important sources of uncertainty affecting our determination of the line position are summarized and quantified in Table I. The largest contribution to the total uncertainty comes from the inaccuracy of the $a b$ initio parameters. In order to quantify this effect we re-peated the multispectrum fits by letting the fixed param-eters of the fit to vary by $20 \%$, with an approach similar to that adopted in Ref. [30]. Another effect taken into ac-count was the temperature dependence of the collisional shift. Since no experimental data are available for these transitions we used ab initio values $(950 \mathrm{kHz} /(\mathrm{atm} \mathrm{K})$ at $300.65 \mathrm{~K}$ ) which determine an uncertainty of $12 \mathrm{kHz}$ for a temperature inaccuracy of $20 \mathrm{mK}$, as it was in our experimental conditions. An additional source of systematic errors is the presence of etalons. We observed a weak etalon effect with a period of $17.1 \mathrm{GHz}$ (created by the opposite facets of one of the cavity mirrors) only for the S(4) transition. In the line-shape analysis we fixed this period while the amplitude and phase were fitted. Its contribution to the uncertainty budget was estimated at the $32 \mathrm{kHz}$ level by repeating the fitting with a period varied by a rather conservative $10 \%$. In Table I we also mention instrumental systematic errors related to the instability of the spectrometer frequency axis and to the pressure gauge nonlinearity. It is worth pointing out that all mentioned uncertainty contributions are $1 \sigma$ values.

\section{COMPARISON WITH THEORY}

The theoretically determined transition frequencies with specified contributions up to $\alpha^{7}[2,3]$ are given in Table II. The details of the calculations can be found in Ref. [2, 3]. These values differ from our experimen-tal results by $2.2 \mathrm{MHz}(2.2 \sigma)$ and $3.3 \mathrm{MHz}(2.9 \sigma)$ for $\mathrm{S}(3)$ and $\mathrm{S}(4)$ transitions, respectively, thus beyond the combined $1 \sigma$ uncertainty level. It should be noted that similar deviations from the theoretical predictions were

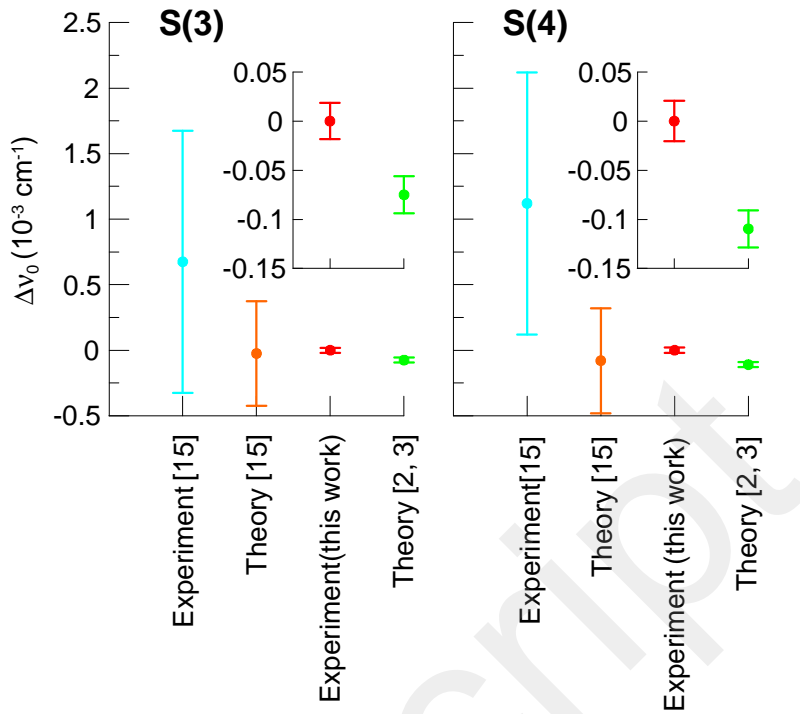

FIG. 3: Comparison of our experimental determinations of the frequencies of the $\mathrm{D}_{2} 2-0 \mathrm{~S}(3)$ and $\mathrm{S}(4)$ transitions with the theoretical ones $[2,3]$ as well as with previous results presented in Ref. [16].

also observed for different kind of experiments on all stable hydrogen isotopologues [30, 32-35]. The reason for this discrepancy has not yet been clarified.

In addition, in Table II and Fig. 3 we compare our results with data available in the literature. Gupta et al. [36] achieved a $0.01 \mathrm{~cm}^{-1}$ uncertainty on the $\mathrm{S}(3)$ transition, which was measured with off-axis integrated cavity output spectroscopy (oa-ICOS). Kassi et al. [16] employed a CRDS technique to determine frequencies of several $\mathrm{D}_{2} 2-0$ transitions, including $\mathrm{S}(3)$ and $\mathrm{S}(4)$ ones. Our results are within their $1 \sigma$ uncertainties which are about 30 times larger than ours. Kassi et al. [16] also reported theoretically determined line positions which occur to be in good agreement with our findings, yet being characterized by a higher uncertainty $(12 \mathrm{MHz})$.

\section{CONCLUSIONS}

In this paper we presented the spectroscopic determination of the frequencies of the $\mathrm{D}_{2} 2-0 \mathrm{~S}(3)$ and $\mathrm{S}(4)$ transitions with the highest accuracy achieved so far. We made use of $a b$ initio quantum scattering calculations for the line-shape analysis to mitigate systematics due to the complex collisional physics involved in the relatively high pressure range explored, from 100 to 900 Torr. Our fi-nal accuracy is $870 \mathrm{kHz}$ and $999 \mathrm{kHz}$ for the $\mathrm{S}(3)$ and $\mathrm{S}(4)$ line positions, respectively, which represents a 30-fold improvement compared to previously available data. As compared to newly calculated line center values exhibiting a $1-\mathrm{MHz}$ level uncertainty, we observe a $2.2 \sigma$ and $2.9 \sigma$ discrepancy for the $\mathrm{S}(3)$ and $\mathrm{S}(4)$ transitions, respectively. The origin of this discrepancy is not yet 
TABLE II: Contributions to the theoretical determination of the energies of the $\mathrm{D}_{2} 2-0 \mathrm{~S}(3)$ and $\mathrm{S}(4)$ transitions [2, 3]. In the bottom part of the table, we also report, for comparison, other available theoretical and experimental data. When unspecified, the uncertainty shall be assumed smaller than the last digit. Note that $1 \mathrm{~cm}^{-1}=29979.2458 \mathrm{MHz}$.

\begin{tabular}{l|c|c}
\hline Contribution & $\mathbf{S}(\mathbf{3})\left(\mathrm{cm}^{-1}\right)$ & $\mathbf{S}(\mathbf{4})\left(\mathrm{cm}^{-1}\right)$ \\
\hline$E^{(2)}$ & 6335.70969 & 6423.95854 \\
$E^{(4)}$ & 0.04180 & 0.04316 \\
$E^{(5)}$ & $-0.03392(2)$ & $-0.03461(2)$ \\
$E^{(6)}$ & -0.00030 & -0.00031 \\
$E^{(7)}$ & $0.00002(1)$ & $0.00002(1)$ \\
$E_{F S}$ & -0.00003 & -0.00003 \\
Total theory [2, 3] & $\mathbf{6 3 3 5 . 7 1 7 2 5 0}(\mathbf{1 9})$ & $\mathbf{6 4 2 3 . 9 6 6 7 7 0}(\mathbf{1 9})$ \\
Experiment (this work) & $\mathbf{6 3 3 5 . 7 1 7 3 2 5}(\mathbf{2 9})$ & $\mathbf{6 4 2 3 . 9 6 6 8 8 0}(\mathbf{3 3})$ \\
Difference & $\mathbf{0 . 0 0 0 0 7 5}(\mathbf{3 5})$ & $\mathbf{0 . 0 0 0 1 1 0}(\mathbf{3 9})$ \\
\hline Theory $[16]$ & $6335.7173(4)$ & $6423.9668(4)$ \\
Experiment $[16]$ & $6335.718(1)$ & $6423.968(1)$ \\
Experiment [36] & $6335.72(1)$ & - \\
\hline
\end{tabular}

fully known.

\section{Acknowledgments}

We thank Krzysztof Pachucki, Jacek Komasa, and Pawel- Czachorowski for providing us with theoretical values of the positions of the $\mathrm{D}_{2} 2-0 \mathrm{~S}(3)$ and $\mathrm{S}(4)$ transitions including all relativistic and QED corrections from Table II. The research is supported by the French-
Polish PHC Polonium program (project 42769ZK for the French part) and by the Polish National Agency for Academic Exchange under the PHC Polonium program (dec. PPN/X/PS/318/2018). S.W . is supported by the Polish Ministry of Science and Higher Education program "Mobility Plus" through Grant No. 1663/MOB/V/2017/0. The contribution of P.W. and H.J. is supported by the National Science Centre, Poland, Projects Nos. 2015/19/D/ST2/02195 and 2018/31/B/ST2/00720.
[1] J. Komasa et al., J. Chem. Theory Comput. 7, 3105 (2011).

[2] P. Czachorowski, M. Puchalski, J. Komasa, and

K. Pachucki, Phys. Rev. A 98, 052506 (2018).

[3] J. Komasa, M. Puchalski, P. Czachorowski, G. - Lach, and K. Pachucki, Phys. Rev. A 100, 032519 (2019).

[4] K. Pachucki and J. Komasa, Phys. Chem. Chem. Phy20, 247 (2018).

[5] K. Pachucki and J. Komasa, Phys. Chem. Chem. Phy21, 10272 (2019).

[6] W. Ubachs, J. Koelemeij, K. Eikema, and E.

SalumMIdesSpectrosc. 320, 1 (2016).

[7] E. J. Salumbides, A. N. Schellekens, B. Gato-Rivera, andW. Ubachs, New J. Phys. 17, 033015 (2015).

[8] E. Salumbides, J. Koelemeij, J. Komasa, K.

Packuctkikema, and W. Ubachs, Phys. Rev. D 87, 112008 (2013).

[9] P. Wcis-lo, H. Tran, S. Kassi, A. Campargue, F. Thibault, and R. Ciury-lo, J. Chem. Phys. 141, 074301 (2014).

[10] P. Wcis- lo,F. Thibault, H. Cybulski, and R. Ciury-lo, Phys. Rev. A 91, 052505 (2015).

[11] P. Wcis-lo,I. E. Gordon, C.-F. Cheng, S.-M. Hu, and R. Ciury-lo, Phys. Rev. A 93, 022501 (2016).

[12] N. H. Ngo, D. Lisak, H. Tran, and J.-M. Hartmann, J. Quant. Spectrosc. Radiat. Transfer 129, 89 (2013).

[13] H. Tran, N. H. Ngo, and J.-M. Hartmann, J. Spectrosc. Radiat. Tra@sfent129, 199 (2013).

[14] P. Wcis- loI. E. Gordon, H. Tran, Y. Tan, S.-M. Hu, A. Campargue, S. Kassi, D. Romanini, C. Hill, R. V.
Kochanov, and L. S. Rothman, J. Quant. Spectrosc. Radiat. Transfer 177, 75 (2016).

[15] M. Konefal- ,M. S- lowin'ski,M. Zaborowski, R. Ciury-lo, D. Lisak, and P. Wcis- lo,J. Quant. Spectrosc. Radiat. Transfer 242, 106784 (2020).

[16] S. Kassi, A. Campargue, K. Pachucki, and J. Komasa, J. Chem. Phys. 136, 184309 (2012).

[17] F. Tauser, A. Leitenstorfer, and W. Zinth, Opt.

Exprids 594 (2003).

[18] J. Burkart, T. Sala, S. Kassi, D. Romanini, and

M. Marangoni, Opt. Lett. 40, 816 (2015).

[19] D. Halmer, G. von Basum, P. Hering, and M.

Mu“Pey. Sci. Instrum. 75, 2187 (2004).

[20] G.-W. Truong, K. O. Douglass, S. E. Maxwell, R. D.

van Zee, D. F. Plusquellic, J. T. Hodges, and D. A. Long, Nature Photonics 7, 532 (2013).

[21] R. Gotti, D. Gatti, P. Mas- lowski,M. Lamperti, M. Belmonte, P. Laporta, and M. Marangoni, J. Chem. Phys. 147, 134201 (2017).

[22] R. H. Dicke, Phys. Rev. 89, 472 (1953).

[23] P. R. Berman, J. Quant. Spectrosc. Radiat. Transfer

12, 1331 (1972).

[24] S. G. Rautian and I. I. Sobelman, Usp. Fiz. Nauk 90, 209 (1966) [Sov. Phys. Usp. 9, 701 (1967)].

[25] D. Priem, F. Rohart, J.-M. Colmont, G. Wlodarczak, and J.-P. Bouanich, J. Mol. Struct. 517, 435 (2000).

[26] M. Nelkin and A. Ghatak, Phys. Rev. 135, A4 (1964).

[27] J. Tennyson et al., Pure Appl. Chem. 86, 1931 (2014).

[28] D. C. Benner, C. P. Rinsland, V. Malathy Devi, M. 
H. Smith, and D. Atkins, J. Quant. Spectrosc. Radiat. Transfer 53, 705 (1995).

[29] F. Thibault, K. Patkowski, P. S. Żuchowski, H. Jóźwiak, R. Ciuryło, and P. Wcisło, J. Quant. Spectrosc. Radiat. Transfer 202, 308 (2017).

[30] P. Wcisło, F. Thibault, M. Zaborowski, S. Wójtewicz, A. Cygan, G. Kowzan, P. Masłowski, J. Komasa, M. Puchalski, K. Pachucki, R. Ciuryło, and D. Lisak, J. Quant. Spectrosc. Radiat. Transfer 213, 41 (2018).

[31] G. Garberoglio, P. Jankowski, K. Szalewicz, and A. H. Harvey, J. Chem. Phys. 137, 154308 (2012).

[32] D. Mondelain, S. Kassi, T. Sala, D. Romanini, D. Gatti, and A. Campargue, J. Mol. Spectrosc. 326, 5 (2016).

[33] M. L. Diouf, F. M. J. Cozijn, B. Darquié, E. J. Salumbides, and W. Ubachs, Opt. Lett. 44, 4733 (2019).

[34] M. Zaborowski, M. Słowiński, K. Stankiewicz, F. Thibault, A. Cygan, H. Jóźwiak, G. Kowzan, P. Masłowski, A. Nishiyama, N. Stolarczyk, S. Wójtewicz, R. Ciuryło, D. Lisak, and P. Wcisło, Opt. Lett. 45, 1603 (2020).

[35] A. Fast and S. A. Meek, arXiv:2002.09333v1.

[36] M. Gupta, T. Owano, D. S. Baer, and A. O'Keefe, Chem. Phys. Lett. 441, 204 (2007). 